\title{
Quantum Law of Motion: Analysis and Extension to Higher Dimensions
}

\author{
A. Bouda* and A. Gharbi ${ }^{\dagger}$ \\ Laboratoire de Physique Théorique, Université de Béjaïa, \\ Route Targa Ouazemour, 06000 Béjaïa, Algeria
}

November 3, 2018

\begin{abstract}
In this paper, we review the recently formulated quantum laws of motion and provide new observations. We also extend these laws to higher dimensions. By applying in two dimensions the obtained relations to charge submitted to an electric central potential, we decide between these laws. Furthermore, we extend the selected law to the relativistic case in higher dimensions.
\end{abstract}

PACS: 03.65.Ca; 03.65.Ta; 04.20.-q

Key words: quantum law of motion, quantum potential, higher dimensions, relativistic extension.

*Electronic address: bouda_a@yahoo.fr

${ }^{\dagger}$ Electronic address: hakimgharbi@yahoo.fr 


\section{Introduction}

Recently, Matone suggested that the quantum potential generates the gravitational potential [1]. This hypothesis was the logical result of the quantum mechanics formulation based on the equivalence postulate [2, 3. In this formulation, it is the quantum Hamilton-Jacobi equation (QHJE) which is investigated. In this context, it is shown that tunnel effect, energy quantization [3, 4] and band structure for the energy spectrum in the Krönig-Penney model [5] directly follow from the QHJE without appealing to the usual axiomatic interpretation of the wave function. Another interesting feature of the QHJE is the fact that the quantum potential, which represents an additional term compared with the classical Hamilton-Jacobi equation (CHJE), can be seen as a term which guarantees the invariance of the Hamilton-Jacobi equation under any coordinate transformation 3. This reminds us of the role played by the gravitational field in general relativity. In this paper, we will review the different quantum laws of motion based on the QHJE. Among them, we can cite the Bohm approach 6] for which Einstein raised a serious objection in the case where the system is described by a real wave function. Einstein's criticism was answered by taking the wave function in the form [2, 3, 7 ,

$$
\phi=\mathrm{R}\left[\alpha \exp \left(i \frac{S_{0}}{\hbar}\right)+\beta \exp \left(-i \frac{S_{0}}{\hbar}\right)\right]
$$

where $\alpha$ and $\beta$ are complex constants. We can also cite Floyd's proposal for which trajectories are obtained by using Jacobi's theorem [8]. However, in this formulation, it seems that there is some confusion in the definition of time parametrization [9, 10, 11]. We essentially devote our discussion to the quantum laws established in 9] and [12.

The paper is organized as follows. In Section 2, we review some features of the Quantum Laws of motion proposed in [9] and [12]. In Section 3, we extend these laws to higher dimensions. In Section 4, we apply in two dimensions the obtained relations to hydrogen atom and then decide between them. In Section 5 , we extend the selected law to the relativistic case in higher dimensions. Section 6 is devoted to conclusion.

\section{The Quantum Laws of Motion}

From the one-dimensional stationary QHJE

$$
\begin{aligned}
& \frac{1}{2 m}\left(\frac{\partial S_{0}}{\partial x}\right)^{2}+ V(x)-E= \\
& \frac{\hbar^{2}}{4 m}\left[\frac{3}{2}\left(\frac{\partial S_{0}}{\partial x}\right)^{-2}\left(\frac{\partial^{2} S_{0}}{\partial x^{2}}\right)^{2}-\left(\frac{\partial S_{0}}{\partial x}\right)^{-1}\left(\frac{\partial^{3} S_{0}}{\partial x^{3}}\right)\right],
\end{aligned}
$$

and by appealing to the coordinate transformation [3, 13 .

$$
x \rightarrow \hat{x} \quad / \quad \frac{\partial \hat{x}}{\partial x}=\frac{\partial S_{0} / \partial x}{\sqrt{2 m(E-V(x))}},
$$


after which the above QHJE takes the classical form,

$$
\frac{1}{2 m}\left(\frac{\partial \hat{S}_{0}(\hat{x})}{\partial \hat{x}}\right)^{2}+\hat{V}(\hat{x})=\hat{E},
$$

it is established in 9] that

$$
\frac{1}{2} \frac{\partial S_{0}}{\partial x} \dot{x}+V(x)=E .
$$

This law, with the use of (2), has allowed to establish the Quantum Newton Law 9 . We first observe that relation (5) itself constitutes a law of motion. In fact, by using in (5) the solution [7, 9, 11] of (2)

$$
S_{0}=\hbar \arctan \left[a \frac{\phi_{1}}{\phi_{2}}+b\right]+\hbar \lambda,
$$

where $\left(\phi_{1}, \phi_{2}\right)$ is a couple of two real independent solutions of the Schrödinger equation (SE), $-\hbar^{2} \phi^{\prime \prime} / 2 m+V \phi=E \phi$, and $(a, b, \lambda)$ are real integration constants satisfying the condition $a \neq 0$, we obtain a first order differential equation representing the quantum law of motion 11 .

Let us now examine the different formulations used to derive relation (5). In [9, we can easily see that (5) is obtained from the Lagrangian

$$
L(x, \dot{x}, a, b)=\frac{1}{2} m \dot{x}^{2}\left(\frac{\partial \hat{x}}{\partial x}\right)^{2}-V(x) .
$$

The non-classical integration constants $a$ and $b$, contained in $\hat{x}$, played the role of hidden parameters. Taking into account relation (3), $L$ and then the resulting quantum law of motion, depend also on the energy $E$. This constant is appeared also after the integration of the obtained equation of motion. It follows that the integration constants are not independent and some of them form a redundant subset [14. Although, this problem is gone round in [10] by using in the Lagrangian formulation the coordinate $\hat{x}, L=L(\hat{x}, \dot{\hat{x}})$, the fact remains that this formulation is not coherent when we use the coordinate $x$.

An analogous remark can be made also in the Hamiltonian formulation. In fact, relation (5) is reproduced in [10] by using the Hamiltonian

$$
H=\frac{P^{2}}{2 m}\left(\frac{\partial x}{\partial \hat{x}}\right)^{2}+V(x),
$$

where $P=\partial S_{0} / \partial x$ is the conjugate momentum. When the canonical equation was applied in [10, it is not taken into account the dependence of the factor $(\partial x / \partial \hat{x})^{2}$ on $E$ and consequently on $H$. This observation was pointed out in the relativistic case in [15]. In order to remedy this weakness, let us rewrite (8) in the following form

$$
P=\sqrt{2 m(H-V)} \frac{\partial \hat{x}}{\partial x} .
$$

With the use of (3) and (6), we can express $\partial \hat{x} / \partial x$ and by applying then (9) we can deduce $P$. Finally, the quantum law of motion can be derived from the canonical equation

$$
\dot{x}=\frac{\partial H}{\partial P}=\left(\frac{\partial P}{\partial H}\right)^{-1} .
$$


This equation leads to the same trajectories as those obtained from Jacobi's theorem, $t-t_{0}=\partial S_{0} / \partial E$, as used by Floyd [8]. In fact, taking the derivative with respect to $x$ of this last relation, we obtain

$$
\frac{d t}{d x}=\frac{\partial^{2} S_{0}}{\partial x \partial E}=\frac{\partial^{2} S_{0}}{\partial E \partial x}=\frac{\partial P}{\partial E}=\frac{\partial P}{\partial H},
$$

which is equivalent to (10). Consequently, when we take into account the dependence on $E$ of $\hat{x}$, as in Floyd's approach, the resulting trajectories will depend on the choice of the solutions of the SE [10] that we will use in the reduced action in order to express $\partial \hat{x} / \partial x$. For the moment, the only way to obtain a coherent Hamiltonian formulation leading to $(5)$ is to use the coordinate system $(\hat{x})$ and to define the conjugate momentum as $\hat{P}=\partial \hat{S}_{0} / \partial \hat{x}$. The transformation to the coordinate system $(x)$ must not be performed until the canonical equation is applied.

The last observation that we make about relation (5) is pointed out in 12 and concerns turning points. At the points where $E=V(x)$, since $\partial S_{0} / \partial x$ never has a vanishing value, we can show from (5) that all the higher temporal derivatives of $x$ take a vanishing value: $\dot{x}=0, \ddot{x}=0, \ldots$ Thus, when the particle gets to one of these points, it can never leave it.

At first glance, the above three observations plead for a new law of motion. That's what is done in [12] where it is proposed a new approach consisting in the construction of a Lagrangian from which we have reproduced the QHJE. This approach was based on the following sensible hypothesis:

1. The Hamilton's principal function is an integral of a Lagrangian.

2. The Lagrangian is a difference between a kinetic term depending on $(x$, $\dot{x}, \ddot{x}, \dot{\ddot{x}})$ and containing the quantum potential, and an external potential.

3. The resulting equation of motion is a fourth order one, in accordance with the one-dimensional QHJE.

In the context of these hypothesis, it is shown that in order to reach the QHJE from the constructed Lagrangian, the condition

$$
\frac{\partial S_{0}}{\partial x}=m \dot{x}
$$

recalling the Bohm relation, is required. Relation (12) represented the new law of motion and it allowed with the use of (2) to establish the modified quantum Newton law 12. Although, (12) reminds us of the classical mechanics, we stress that it describes the quantum motion because $\partial S_{0} / \partial x$ represents the solution of the QHJE, Eq. (2). At first glance, this model seems attractive. However, as we will see in Section 4, Eq. (12) leads to a deadlock when we apply its twodimensional version to the motion of a charge submitted to an electric central potential.

\section{Extension to Higher Dimensions}

Although relation (12) has the same form as Bohm's law of motion, it is fundamentally different. In fact, in contrast to Bohm's theory, the reduced action 
$S_{0}$ in (12) is related to the wave function by (1) and therefore it never takes a constant value even in the case where the wave function is real, up to a constant phase factor. As in Bohm's theory, the extension of relation (12) to three dimensions can be sensibly assumed as

$$
m \dot{x}=\frac{\partial S_{0}}{\partial x}, \quad m \dot{y}=\frac{\partial S_{0}}{\partial y}, \quad m \dot{z}=\frac{\partial S_{0}}{\partial z} .
$$

Concerning relation (5), before we extend it to higher dimensions, it is instructive to reproduce it in one dimension with a novel approach which we will use to perform this extension.

As $\hat{S}_{0}(\hat{x})=S_{0}(x), \hat{V}(\hat{x})=V(x)$ and $\hat{E}=E$ [3, 9, 13], relation (4) can be written as

$$
\frac{1}{2 m}\left(\frac{\partial S_{0}(x)}{\partial x}\right)^{2}\left(\frac{\partial x}{\partial \hat{x}}\right)^{2}+V(x)=E .
$$

On the other hand, if we use the coordinate system $(\hat{x})$ in which the quantum potential is canceled, the conjugate momentum takes the classical form

$$
\frac{\partial \hat{S}_{0}}{\partial \hat{x}}=m \dot{\hat{x}}
$$

from which we deduce that

$$
\frac{\partial S_{0}}{\partial x}\left(\frac{\partial x}{\partial \hat{x}}\right)^{2}=m \dot{x}
$$

Substituting this expression in (14), we straightforwardly get to relation (5).

In what follows, we will use Einstein's convention for repeated indexes. Let us consider in a $D$-dimensional space the QHJE

$$
\delta^{i j} \frac{1}{2 m} \frac{\partial S_{0}}{\partial x^{i}} \frac{\partial S_{0}}{\partial x^{j}}-\delta^{i j} \frac{\hbar^{2}}{2 m R} \frac{\partial^{2} R}{\partial x^{i} \partial x^{j}}+V\left(x^{i}\right)=E,
$$

where the functions $R$ and $S_{0}$ satisfy the continuity equation

$$
\delta^{i j} \frac{\partial}{\partial x^{i}}\left(R^{2} \frac{\partial S_{0}}{\partial x^{j}}\right)=0,
$$

and $\delta^{i j}$ is the Kronecker symbol. In the coordinate system $\left(\hat{x}^{i}\right)$ in which the quantum potential is canceled, the QHJE takes the classical form

$$
\delta^{i j} \frac{1}{2 m} \frac{\partial \hat{S}_{0}}{\partial \hat{x}^{i}} \frac{\partial \hat{S}_{0}}{\partial \hat{x}^{j}}+\hat{V}\left(\hat{x}^{i}\right)=\hat{E} .
$$

As $\hat{S}_{0}\left(\hat{x}^{i}\right)=S_{0}\left(x^{i}\right), \hat{V}\left(\hat{x}^{i}\right)=V\left(x^{i}\right)$ and $\hat{E}=E$, relation (19) turns out to be

$$
\delta^{i j} \frac{1}{2 m} \frac{\partial S_{0}}{\partial x^{l}} \frac{\partial x^{l}}{\partial \hat{x}^{i}} \frac{\partial S_{0}}{\partial x^{k}} \frac{\partial x^{k}}{\partial \hat{x}^{j}}+V\left(x^{i}\right)=E .
$$

As in general relativity, we assume that the coordinate system $\left(\hat{x}^{i}\right)$, in which the laws of motion take classical forms, is locally flat. Therefore, we have

$$
\frac{\partial \hat{S}_{0}}{\partial \hat{x}^{i}}=m \frac{d \hat{x}_{i}}{d t}=m \delta_{i l} \frac{d \hat{x}^{l}}{d t}
$$


from which we deduce that

$$
\frac{\partial S_{0}}{\partial x^{l}} \frac{\partial x^{l}}{\partial \hat{x}^{i}}=m \delta_{i l} \frac{\partial \hat{x}^{l}}{\partial x^{n}} \dot{x}^{n} .
$$

Multiplying each side of this last relation by $\delta^{i j} \partial x^{k} / \partial \hat{x}^{j}$, we obtain

$$
\begin{aligned}
\delta^{i j} \frac{\partial S_{0}}{\partial x^{l}} \frac{\partial x^{l}}{\partial \hat{x}^{i}} \frac{\partial x^{k}}{\partial \hat{x}^{j}} & =m \delta^{i j} \delta_{i l} \frac{\partial x^{k}}{\partial \hat{x}^{j}} \frac{\partial \hat{x}^{l}}{\partial x^{n}} \dot{x}^{n} \\
& =m \delta_{l}^{j} \frac{\partial x^{k}}{\partial \hat{x}^{j}} \frac{\partial \hat{x}^{l}}{\partial x^{n}} \dot{x}^{n} \\
& =m \frac{\partial x^{k}}{\partial \hat{x}^{j}} \frac{\partial \hat{x}^{j}}{\partial x^{n}} \dot{x}^{n} \\
& =m \frac{\partial x^{k}}{\partial x^{n}} \dot{x}^{n} \\
& =m \delta_{n}^{k} \dot{x}^{n} \\
& =m \dot{x}^{k} .
\end{aligned}
$$

Using this result in (20), we find

$$
\frac{1}{2} \frac{\partial S_{0}}{\partial x^{k}} \dot{x}^{k}+V\left(x^{i}\right)=E .
$$

This represents the higher dimension version of relation (5). Although, relation (24) works in classical mechanics $\left(\partial S_{0}^{\text {clas }} / \partial x^{k}=m \dot{x}_{k}\right)$, it describes the quantum motion because in (24) $\partial S_{0} / \partial x^{k}$ is the solution in higher dimensions of the QHJE, already investigated in [16. The problem of the immobility of particles at turning points disappears. In fact, when the space dimension is higher than one, we cannot show from $(24)$ that the derivatives $\dot{x}^{k}, \ddot{x}^{k}, \ldots(k=1,2, \ldots, D)$ take simultaneously vanishing values.

\section{Hydrogen atom in two dimensions}

In this Section, let us apply in two dimensions the laws of motion (13) and (24) to the hydrogen atom for which the potential is

$$
V(r)=-\frac{e^{2}}{r},
$$

where $e^{2}=q^{2} / 4 \pi \epsilon_{0}, q$ being the absolute value of the electron charge. By using polar coordinates $(r, \theta)$ and writing $\psi(r, \theta)=R(r) \Theta(\theta)$, it is well known that Schrödinger's equation

$$
-\frac{\hbar^{2}}{2 m} \Delta \psi+V(r) \psi=E \psi,
$$

leads to the two following separated relations

$$
\frac{d^{2} R}{d \rho^{2}}+\frac{1}{\rho} \frac{d R}{d \rho}+\left[\frac{2}{\rho}-\frac{l^{2}}{\rho^{2}}-\alpha^{2}\right] R=0
$$

and

$$
\frac{d^{2} \Theta}{d \theta^{2}}+l^{2} \Theta=0
$$


where $l$ is an integration constant, $\rho=r / a_{0}$ and $\alpha^{2}=-E / E_{I}, a_{0}$ and $E_{I}$ being respectively Bohr's atomic radius $\left(a_{0}=\hbar^{2} / m e^{2}\right)$ and the ionization energy $\left(E_{I}=m e^{4} / 2 \hbar^{2}\right)$ of the Hydrogen atom. Choosing for (28) as independent real solutions the two following functions

$$
\Theta_{1}=\cos l \theta, \quad \Theta_{2}=\sin l \theta,
$$

and imposing the conditions

$$
\Theta_{1}(\theta)=\Theta_{1}(\theta+2 \pi), \quad \Theta_{2}(\theta)=\Theta_{2}(\theta+2 \pi),
$$

we deduce that $l$ must be an integer number. In [17, it is shown that a physical solution for $(27)$ is

$$
R_{1}(\rho)=\rho^{|l|} \exp (-\alpha \rho) L_{n-|l|}^{2|l|}(2 \alpha \rho)
$$

where $L_{s}^{k}$ are the generalized Laguerre polynomials, $n=0,1,2,3 \ldots$ is the principal quantum number $(-n \leq l \leq n)$ and

$$
\alpha=\frac{1}{n+1 / 2} \text {. }
$$

This last relation leads to

$$
E(n) \equiv E_{n}=-\frac{E_{I}}{(n+1 / 2)^{2}} .
$$

A second real independent solution $R_{2}(\rho)$ for $(27)$ can be derived by using the Wronskian $W\left(R_{1}, R_{2}\right)$ [18]

$$
R_{2}(\rho)=R_{1}(\rho) \int \frac{\exp \left[-\int \frac{d \rho}{\rho}\right]}{R_{1}^{2}(\rho)} d \rho=R_{1}(\rho) \int \frac{d \rho}{\rho R_{1}^{2}(\rho)} .
$$

As shown in [16], the reduced action in two dimensions is

$$
S_{0}=\hbar \arctan \left[\frac{R_{1} \Theta_{1}+\nu_{2} R_{1} \Theta_{2}+\nu_{3} R_{2} \Theta_{1}+\nu_{4} R_{2} \Theta_{2}}{\mu_{1} R_{1} \Theta_{1}+\mu_{2} R_{1} \Theta_{2}+\mu_{3} R_{2} \Theta_{1}+R_{2} \Theta_{2}}\right]+\hbar \lambda,
$$

where $\left(\nu_{2}, \nu_{3}, \nu_{4}, \mu_{1}, \mu_{2}, \mu_{3}, \lambda\right)$ are real integration constants. For the ground state $(n=0, l=0)$, by (32) we have $\alpha=2$. As $L_{0}^{0}=1$ and $\rho=r / a_{0}$, with the use of (29), (31) and (34), we deduce that

$$
\Theta_{1}=1, \quad \Theta_{2}=0,
$$

and

$$
R_{1}=\exp \left(-\frac{2 r}{a_{0}}\right), \quad R_{2}=\exp \left(-\frac{2 r}{a_{0}}\right) \int_{r_{0}}^{r} \frac{\exp \left(4 r^{\prime} / a_{0}\right)}{r^{\prime}} d r^{\prime},
$$

Note that the lower boundary $r_{0}$ can be arbitrary chosen. Therefore, in order to avoid the singular point $r^{\prime}=0$ in (37), we choose $r_{0}$ positive. Note also that with suitable integration boundaries, the integral in (37) can be identified to the exponential integral $\mathbf{E i}(\mathrm{r})$ defined as the Cauchy's principal value of

$$
\operatorname{Ei}(x)=\int_{-\infty}^{x} \frac{\exp (t)}{t} d t \quad(x>0) .
$$


It follows that for the ground state, expression (35) reduces to

$$
S_{0}=\hbar \arctan \left[\frac{R_{1}+\nu_{3} R_{2}}{\mu_{1} R_{1}+\mu_{3} R_{2}}\right]+\hbar \lambda,
$$

where $R_{1}(r)$ and $R_{2}(r)$ are given in (37).

In polar coordinates, the law of motion (13) takes the form

$$
\frac{\partial S_{0}}{\partial r}=m \dot{r}, \quad \frac{\partial S_{0}}{\partial \theta}=m r^{2} \dot{\theta},
$$

while the law of motion (24) turns out to be

$$
\dot{r} \frac{\partial S_{0}}{\partial r}+\dot{\theta} \frac{\partial S_{0}}{\partial \theta}=2[E-V(r)] .
$$

Substituting (39) in (40), we get to

$$
\begin{aligned}
\hbar\left(\mu_{1} \nu_{3}-\mu_{3}\right)\left[\frac{2}{a_{0}} \exp \left(-\frac{4 r}{a_{0}}\right) \int_{r_{0}}^{r} \frac{\exp \left(4 r^{\prime} / a_{0}\right)}{r^{\prime}} d r^{\prime}+\right. \\
\left.\frac{1}{r} \exp \left(\frac{2 r}{a_{0}}\right)\right]=m \dot{r} H(r),
\end{aligned}
$$

and

$$
m r^{2} \dot{\theta}=0,
$$

where $H(r)=\left(R_{1}+\nu_{3} R_{2}\right)^{2}+\left(\mu_{1} R_{1}+\mu_{3} R_{2}\right)^{2}$. However, with the use of (25) and (33), and by substituting (39) in (41), we get to

$$
\begin{aligned}
\hbar\left(\mu_{1} \nu_{3}-\mu_{3}\right) \dot{r}\left[\frac{2}{a_{0}} \exp \left(-\frac{4 r}{a_{0}}\right) \int_{r_{0}}^{r} \frac{\exp \left(4 r^{\prime} / a_{0}\right)}{r^{\prime}} d r^{\prime}+\right. \\
\left.\frac{1}{r} \exp \left(\frac{2 r}{a_{0}}\right)\right]=2 H(r)\left[-\frac{2 m e^{4}}{\hbar^{2}}+\frac{e^{2}}{r}\right] .
\end{aligned}
$$

Relation (43) results from (13) and indicates that $\theta$ is constant. This result is unacceptable and forced us to abandon the law of motion (13). Relation (44) results from (24) and does not allow to determine $\theta$. This indicates that relation (24) does not ensure a complete description of the quantum motion when the number of degrees of freedom is higher than one. However, this is not sufficient to reject relation (24). In fact, even in classical mechanics, it is well-known that the law of energy conservation does not allow to determine completely the motion except for systems of one degree of freedom. This feature encourage us to pay attention to relation (5) and its extended version (24).

\section{The Relativistic Case}

Before we establish the relativistic version of (24), it is instructive to remind us of the following main points:

1. When the coordinate system $(\hat{x})$ is used to apply Jacobi's theorem 9 or to express the Lagrangian in order to obtain the equation of motion [10, relation (5) is derived without any mathematical ambiguity. It is also the case for the Hamiltonian formulation. 
2. In the coordinate system $(\hat{x})$, the QHJE and the other motion laws take the classical forms meaning that the choice of this system is made in such a way as to cancel the effect of the quantum potential. This strongly reminds us of the equivalence principle of general relativity which allows to admit the existence of a coordinate system in which the gravitational field is locally canceled.

3. Compared to the CHJE, the quantum potential is an additional term which guarantees the covariance of the QHJE [3]. The same role is also played by the gravitational field in general relativity.

4. The gravitational potential is generated by the quantum potential 1 .

Unquestionably, the above remarks plead in favor of (5) and (24). Consequently, if we would like to continue to believe in the link between the quantum potential and the gravitational field, we have a further argument to abandon the quantum law (12) and also the hypothesis, though attractive, enumerated in Section 2 and which allowed in 12 to establish (12). Concerning the reservations expressed in Section 2 about (5), we can make the following observations. The absence for the moment of a coherent Lagrangian or Hamiltonian formulation in any system coordinate, except in $(\hat{x})$ in which the quantum potential is canceled, must not imply the rejection of the approach. With regard to the particle immobility at turning points, pointed out in [12, this problem appears only in one dimension. As we have seen in Section 3, this problem disappears in realistic models for which the space dimension is higher than one.

Let us now establish the relativistic version of (24). In the context of the equivalence postulate, the $D$-dimensional stationary relativistic QHJE for spinless system reads 19

$$
\delta^{i j} \frac{1}{2 m} \frac{\partial S_{0}}{\partial x^{i}} \frac{\partial S_{0}}{\partial x^{j}}-\delta^{i j} \frac{\hbar^{2}}{2 m R} \frac{\partial^{2} R}{\partial x^{i} \partial x^{j}}+\frac{m^{2} c^{4}-\left[E-V\left(x^{i}\right)\right]^{2}}{2 m c^{2}}=0,
$$

where the functions $R$ and $S_{0}$ satisfy the continuity equation

$$
\delta^{i j} \frac{\partial}{\partial x^{i}}\left(R^{2} \frac{\partial S_{0}}{\partial x^{j}}\right)=0 .
$$

The summation on $i$ and $j$ does not concern the time component [3. In the coordinate system $\left(\hat{x}^{i}\right)$ in which the quantum potential is canceled, the relativistic QHJE takes the classical form

$$
\delta^{i j} \frac{1}{2 m} \frac{\partial \hat{S}_{0}}{\partial \hat{x}^{i}} \frac{\partial \hat{S}_{0}}{\partial \hat{x}^{j}}+\frac{m^{2} c^{4}-\left[\hat{E}-\hat{V}\left(\hat{x}^{i}\right)\right]^{2}}{2 m c^{2}}=0 .
$$

As $\hat{S}_{0}\left(\hat{x}^{i}\right)=S_{0}\left(x^{i}\right), \hat{V}\left(\hat{x}^{i}\right)=V\left(x^{i}\right)$ and $\hat{E}=E$, relation (47) turns out to be

$$
\delta^{i j} \frac{1}{2 m} \frac{\partial S_{0}}{\partial x^{l}} \frac{\partial x^{l}}{\partial \hat{x}^{i}} \frac{\partial S_{0}}{\partial x^{k}} \frac{\partial x^{k}}{\partial \hat{x}^{j}}+\frac{m^{2} c^{4}-\left[E-V\left(x^{i}\right)\right]^{2}}{2 m c^{2}}=0 .
$$

On the other hand, the coordinate system $\left(\hat{x}^{i}\right)$ being locally flat, the conjugate momentum takes the following classical relativistic form

$$
\frac{\partial \hat{S}_{0}}{\partial \hat{x}^{i}}=m \frac{d \hat{x}_{i}}{d \tau}=m \delta_{i l} \frac{d \hat{x}^{l}}{d \tau}
$$


where $d \tau$ is an element of the proper time associated to the particle. Relation (49) can be obtained from (21) by substituting $d t$ by $d \tau$. Thus, in the same manner as in Section 3, we can show from (49) that

$$
\delta^{i j} \frac{\partial S_{0}}{\partial x^{l}} \frac{\partial x^{l}}{\partial \hat{x}^{i}} \frac{\partial x^{k}}{\partial \hat{x}^{j}}=m \frac{d x^{k}}{d \tau} .
$$

Using this result in (48), we find

$$
\frac{\partial S_{0}}{\partial x^{k}} \frac{d x^{k}}{d \tau}+\frac{m^{2} c^{4}-\left[E-V\left(x^{i}\right)\right]^{2}}{m c^{2}}=0 .
$$

This represents the relativistic quantum law of motion in higher dimensions. Although, relation (51) works in classical relativistic mechanics $\left(\partial S_{0}^{\text {clas } / r e l a t} / \partial x^{k}\right.$ $\left.=m d x_{k} / d \tau\right)$, it describes the relativistic quantum motion because in (51) $\partial S_{0} / \partial x^{k}$ represents the solution of the relativistic QHJE, Eq. (45). As in the non-relativistic case [16], one can check that the solutions of (45) and (46) are

$$
S_{0}=\hbar \arctan \left(\frac{\phi_{1}}{\phi_{2}}\right)+\hbar l,
$$

and

$$
R=k \sqrt{\phi_{1}^{2}+\phi_{2}^{2}},
$$

where $\phi_{1}$ and $\phi_{2}$ are two real independent solutions of the stationary KleinGordon equation,

$$
-\frac{\hbar^{2}}{2 m} \Delta \phi+\frac{m^{2} c^{4}-\left[E-V\left(x^{i}\right)\right]^{2}}{2 m c^{2}} \phi=0,
$$

$l$ and $k$ arbitrary integration constants, and $\Delta$ the $D$-dimensional Laplacian. We mention that in the separated variable case, it is possible to make explicit in (52) all the integration constants, as in the non-relativistic case [16].

Finally, we indicate that in one dimension, relation (51) reproduces the same result as the one obtained in [15]. In fact, (51) allows us to write

$$
\frac{\partial S_{0}}{\partial x} \frac{d x}{d \tau}+\frac{m^{2} c^{4}-[E-V(x)]^{2}}{m c^{2}}=0 .
$$

Multiplying by $d \tau / d t$, we have

$$
\frac{\partial S_{0}}{\partial x} \frac{d x}{d t}+\frac{m^{2} c^{4}-[E-V(x)]^{2}}{m c^{2}} \frac{d \tau}{d t}=0 .
$$

On the other hand, the time component being not concerned by the coordinate transformation $(\hat{t}=t)[3$, in the system $(\hat{x})$ where the space is locally flat, we have

$$
d \tau^{2}=d t^{2}-\frac{1}{c^{2}} d \hat{x}^{2}=d t^{2}-\frac{1}{c^{2}}\left(\frac{\partial \hat{x}}{\partial x}\right)^{2} d x^{2} .
$$

By substituting in this last relation the expression

$$
\left(\frac{\partial \hat{x}}{\partial x}\right)^{2}=c^{2} \frac{\left(\partial S_{0} / \partial x\right)^{2}}{[E-V(x)]^{2}-m^{2} c^{4}},
$$


which defines the transformation $x \rightarrow \hat{x}$ allowing to cancel the quantum potential [15, we get to

$$
\left(\frac{d \tau}{d t}\right)^{2}=\frac{[E-V(x)]^{2}-m^{2} c^{4}-\dot{x}^{2}\left(\partial S_{0} / \partial x\right)^{2}}{[E-V(x)]^{2}-m^{2} c^{4}}
$$

Using this expression in (56), we reproduce the one-dimensional relativistic quantum law of motion,

$$
\frac{\partial S_{0}}{\partial x} \frac{d x}{d t}+\frac{m^{2} c^{4}-[E-V(x)]^{2}}{E-V(x)}=0,
$$

already obtained in [15.

\section{Conclusion}

Before summarizing the main results of the present investigation, let us come back to the quantum law (5) established in [9]. Despite its present insufficiencies observed in Section 2, we think that it is useful to investigate this law and its extended versions in more detail for the following reasons.

1. The problem of the particle immobility at turning points is specific to the one-dimensional space. In a realistic model, we cannot ignore the other dimensions of the space and this problem disappears.

2. The fact of the absence for the moment of a coherent Lagrangian or Hamiltonian formulation which works in any coordinate system does not mean that law (5) must be rejected. In fact, in the same manner as for the law of motion in general relativity, relation (5) is rigorously established in the particular system $(\hat{x})$ in which the quantum potential is canceled. The transformation from $(\hat{x})$ to another system $(x)$, which can be for example the laboratory frame, is performed after the equation of motion is obtained in the system $(\hat{x})$.

3. Another interesting feature of (5) is the nodal structure of the quantum trajectories which follow from it. In fact, it is shown in 11] that to each classical trajectory there is a family of quantum trajectories which all pass through some points constituting nodes and belonging to the classical trajectory. In addition, there is an interesting relation between de Broglie's wavelength and the length separating adjacent nodes which become infinitely close in the limit $\hbar \rightarrow 0$. Also, it is shown [1] that in the classical limit all the quantum trajectories tend to be identical to the classical one.

Furthermore, the manner in which the quantum law (5) is derived allows to establish a parallel between the postulate equivalence of quantum mechanics and the one of general relativity. This suggests that relation (5) and its extended versions may play an important role in the search for a possible link, already investigated by Matone [1] and Carroll [20, between the quantum potential and the gravitational potential.

To summarize, it is in this spirit that we have performed in Sections 3 and 5 the extension to higher dimensions of relation (5) respectively in the 
non-relativistic case and the relativistic one. We have also applied its twodimensional version in the non-relativistic case to the hydrogen atom. An interesting question is how to complete relations (24) and (51) in order to describe in its entirety the quantum motion in any dimension.

\section{References}

1. M. Matone, Found. Phys. Lett. 15 (2002) 311.

2. A.E. Faraggi and M. Matone, Phys. Lett. B450 (1999) 34; Phys. Lett. B437 (1998) 369.

3. A.E. Faraggi and M. Matone, Int. J. Mod. Phys. A15 (2000) 1869.

4. A.E. Faraggi and M. Matone, Phys. Lett. B445 (1999) 357.

5. A. Bouda and A. Mohamed Meziane, Int. J. Theo. Phys. 45 (2006) 2377.

6. D. Bohm, Phys. Rev. 85 (1952) 166; Phys. Rev. 85 (1952) 180; Phys. Rev. 89 (1953) 458; D. Bohm and J.P. Vigier, Phys. Rev. 96 (1954) 208.

7. A. Bouda, Found. Phys. Lett. 14 (2001) 17.

8. E.R. Floyd, Phys. Rev. D26 (1982) 1339; quant-ph/0009070.

9. A. Bouda and T. Djama, Phys. Lett. A285 (2001) 27.

10. A. Bouda and T. Djama, Phys. Lett. A296 (2002) 312.

11. A. Bouda and T. Djama, Physica Scripta 66 (2002) 97.

12. A. Bouda, Int. J. Mod. Phys. A18 (2003) 3347.

13. A.E. Faraggi and M. Matone, Phys. Lett. A249 (1998) 180.

14. E.R. Floyd, private communication.

15. A. Bouda and F. Hammad, Acta Physica Slovaca 52 (2002) 101.

16. A. Bouda and A. Mohamed Meziane, Int. J. Theo. Phys. 45 (2006) 1323.

17. D.GW. Parfitt and M.E. Portnoi, J. Math. Phys. 43 (2002) 4681; X.L. Yang, S.H. Guo, F.T. Chan, K.W. Wong and W.Y. Ching, Phys. Rev. A43 (1991) 1186.

18. N. Piskounov, Calcul Différentiel et intégral, Tome II, Edition Mir, Moscou (1980).

19. G. Bertoldi, A.E. Faraggi and M. Matone, Class. Quant. Grav. 17 (2000) 3965 .

20. R. Carroll, gr-qc/0406004. 